\title{
Correction to: FLT3 inhibitor lestaurtinib plus chemotherapy for newly diagnosed KMT2A-rearranged infant acute lymphoblastic leukemia: Children's Oncology Group trial AALL0631
}

Patrick A. Brown (D) - John A. Kairalla · Joanne M. Hilden · ZoAnn E. Dreyer • Andrew J. Carroll • Nyla A. Heerema • Cindy Wang · Meenakshi Devidas • Lia Gore • Wanda L. Salzer • Naomi J. Winick (D) William L. Carroll •

Elizabeth A. Raetz • Michael J. Borowitz • Donald Small • Mignon L. Loh · Stephen P. Hunger (D)

Published online: 12 April 2021

(c) The Author(s), under exclusive licence to Springer Nature Limited 2021

Correction to: Leukemia

https://doi.org/10.1038/s41375-021-01177-6
Figure 3 of the original article was an incorrect version. The correct figure is:

The original article has been updated.
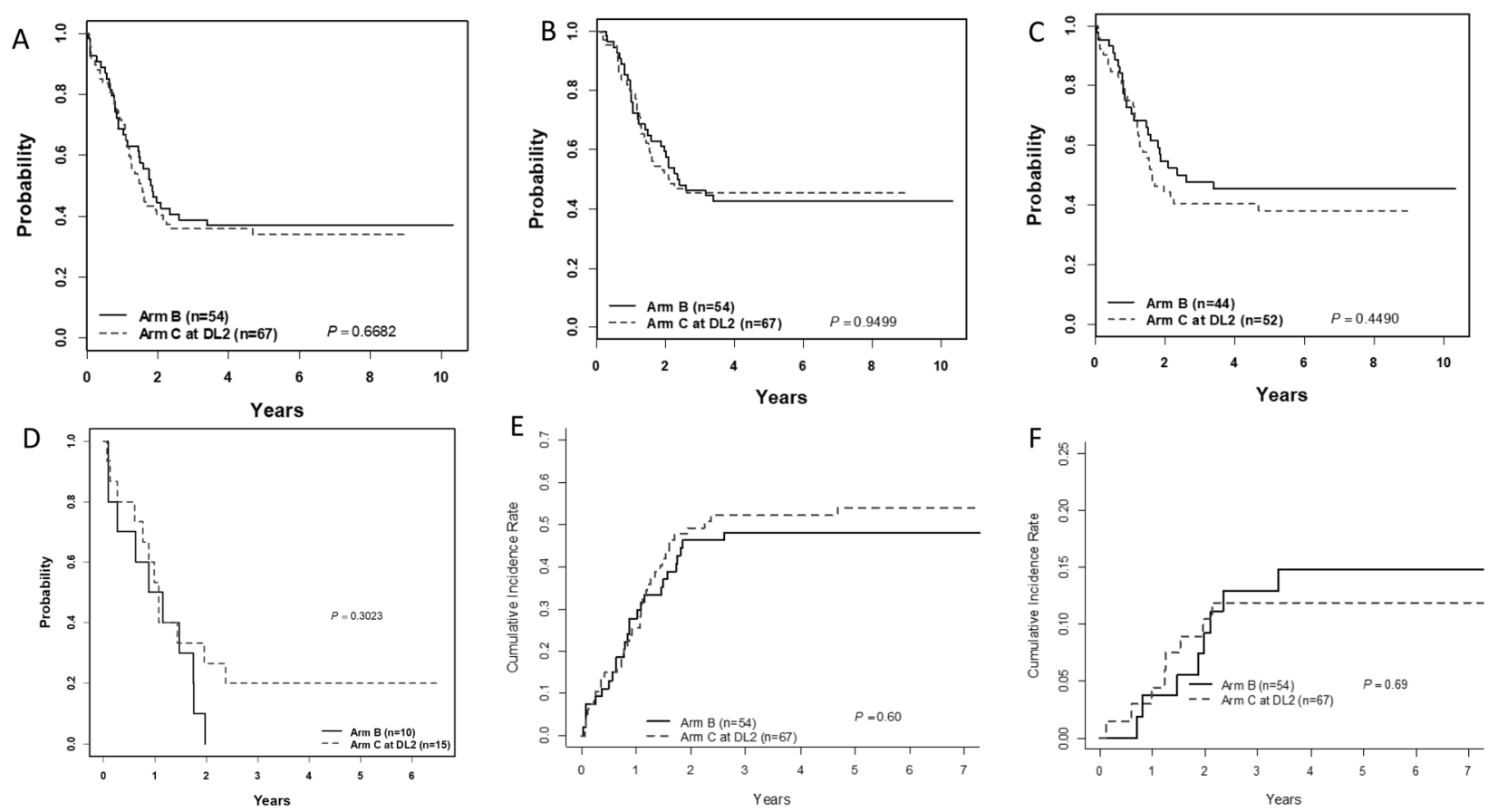

Fig. 3 